\title{
Internal Fixation of Pauwels Type-3 Undisplaced- incomplete Insufficiency Femoral Neck Fractures with Cephalomedullary Nails
}

\author{
Joo-Hyoun Song, MD, PhD, Jongmin Kim, MD, Seungbae Oh, MD \\ Department of Orthopedic Surgery, The Catholic University of Korea, St. Vincent Hospital, Suwon, Korea
}

Femoral neck stress fractures are rare and when treating are difficult to achieve favorable outcomes. This study characterizes outcomes associated with the use of cephalomedullary nails for fixation of Pauwels type-3 vertical femoral neck undisplaced-incomplete insufficiency fractures. Four consecutive patients with a Pauwels type-3 vertical femoral neck tensile insufficiency fracture from 2016 to 2018 were reviewed. Magnetic resonance imaging data revealed tensile visible fracture lines and hip-joint effusions with a high shear angle. For all patients, bone mineral density and vitamin D levels were low; vitamin D therapy was initiated immediately. Surgical procedures were conducted with cephalomedullary nails (Gamma 3 locking nail system; Stryker) under general anesthesia. A cephalomedullary nail appears to be a safe and effective alternative to the use of multiple parallel screws or a sliding hip screw for fixation of vertical femoral neck stress fractures (level of evidence: Level V).

Key Words: Femur Neck, Fractures, Stress, Osteomalacia, Nails

Femoral neck stress fractures are relatively rare and may present as sports-related injuries. Atraumatic femoral neck stress fractures are particularly uncommon (5\% of all stress fractures) and prevalent among athletes and military recruits; women are at higher risk in both populations, with an overall

Submitted: September 17, 2019 1st revision: November 25, 2019 Final acceptance: December 19, 2019

Address reprint request to

Seungbae Oh, MD

(https://orcid.org/0000-0001-8523-9990)

Department of Orthopaedic Surgery, The Catholic University of

Korea, St. Vincent's Hospital, 93 Jungbu-daero, Paldal-gu, Suwon

16247, Korea

TEL: +82-31-249-8966 FAX: +82-31-254-7186

E-mail: minerba5ahanmail.net

This is an Open Access article distributed under the terms of the Creative Commons Attribution Non-Commercial License (http://creativecommons. org/licenses/by-nc/4.0) which permits unrestricted non-commercial use, distribution, and reproduction in any medium, provided the original work is properly cited. incidence of stress fractures of $9.2 \%$ (vs. $3 \%$ for males) in the military and $9.7 \%$ (vs. $6.5 \%$ for males) in athletes ${ }^{1}$. These injuries may also more commonly occur in individuals with underlying metabolic disorders. Bone mineral density (BMD) is usually decreased in patients with femoral neck stress fractures.

The majority of femoral neck fractures are incomplete and undisplaced, therefore, early diagnosis with appropriate imaging in patients with a history of groin pain might be crucial in detecting them at early stages. Prompt diagnosis facilitates the earliest return to pre-morbid functional activity levels. Initial magnetic resonance imaging (MRI) screening is important for predicting fracture progression requiring surgical intervention ${ }^{2}$.

Differentiating between fatigue and insufficiency typestress fractures is important. Fatigue fractures typical manifest as overuse stress fractures and occur predominantly in athletes and military recruits with normal bone density. Insufficiency fractures are most commonly observed in 


\section{Hip \& Pelvis}

Hip Pelvis 32(2): 105-111, 2020

those with low BMD (e.g., runners, individuals with metabolic bone disease or osteoporosis). Fatigue fractures are typically observed in normal bone under excessive or abnormal strain, while insufficiency fractures occur in abnormal bone under normal strain.

Osteomalacia-a bone metabolic disorder associated with defective bone mineralization-is a very common cause of femoral insufficiency type-stress fractures. Osteomalacia ultimately results from a vitamin D deficiency ${ }^{3)}$ and results in softening of the bones leading to the potential production of a focal concentration of stresses in the femoral neck. This fact emphasizes the contribution of 25-hyroxyvitiamin D (25 (OH) D) deficiency to bone demineralization, and its role in the pathogenesis of osteomalacia, which may be an underappreciated disease $e^{4)}$. In insufficiency fractures, osteomalacia may be the most probable underlying etiology.

When considering surgery, femoral neck insufficiency fractures with increasingly vertically oriented fracture lines may experience more shear force; while cannulated screws may be inadequate for fixation under these circumstances, fixed-angle devices (e.g., cephalomedullary nails, dynamic hip screws) may be superior ${ }^{5,6}$. However, few studies have provided treatment guidelines for internal fixation of femoral neck stress fractures.

The purpose of this study is to report a case series of vertically oriented femoral neck undisplaced-incomplete insufficiency fractures treated with cephallomedullary nails to highlight the importance of: i) early suspicion of this injury, even in normal individuals, ii) providing the right treatment, and iii) avoiding possible complications.

\section{CASE REPORT}

Five consecutive patients admitted to our institution between 2016 and 2018 with undisplaced-incomplete insufficiency fractures of the femoral neck were identified. Cases were considered positive if visible tensile fracture lines and marrow edema were visible upon MRI. One patient refused surgery and the remaining four underwent internal fixation with a cephalomedullary nail (Gamma 3 locking nail system; Stryker, Kalamazoo, MI, USA).

This study has been reviewed and approved by the Institutional Review Board (IRB) of the St. Vincent's Hospital (IRB No. VC19ZESI0025). Also, informed consent was obtained from all the individuals participating in this study.

Inclusion criteria for this case series individuals of any age or sex who had sustained an undisplaced-incomplete insufficiency fracture of the femoral neck. Pauwels type3 vertical femoral neck tension-type insufficiency fractures,

Table 1. Demographics of Patients

\begin{tabular}{lc}
\hline \hline Variable & Value \\
\hline Age (yr) & $61.8(20-82)$ \\
Sex (male/female) & $1 / 3$ \\
BMI (kg/m²) & $21.3(17.6-27.2)$ \\
Operative side (right/left) & $3 / 1$ \\
BMD (Z-score) femur total & $-1.85(-2.9--1.3)$ \\
BMD (Z-score) femur neck & $-1.60(-2.2--1.2)$ \\
Pauwels angle ( $\left.{ }^{\circ}\right)$ & $79.8(76.0-83.1)$ \\
Duration between initial & $12(5-21)$ \\
symptoms and fracture (day) & $60(38-80)$ \\
\hline Duration of surgery (min) & \\
\hline
\end{tabular}

Values are presented as mean (range) or number only. BMI: body mass index, BMD: bone mineral density.
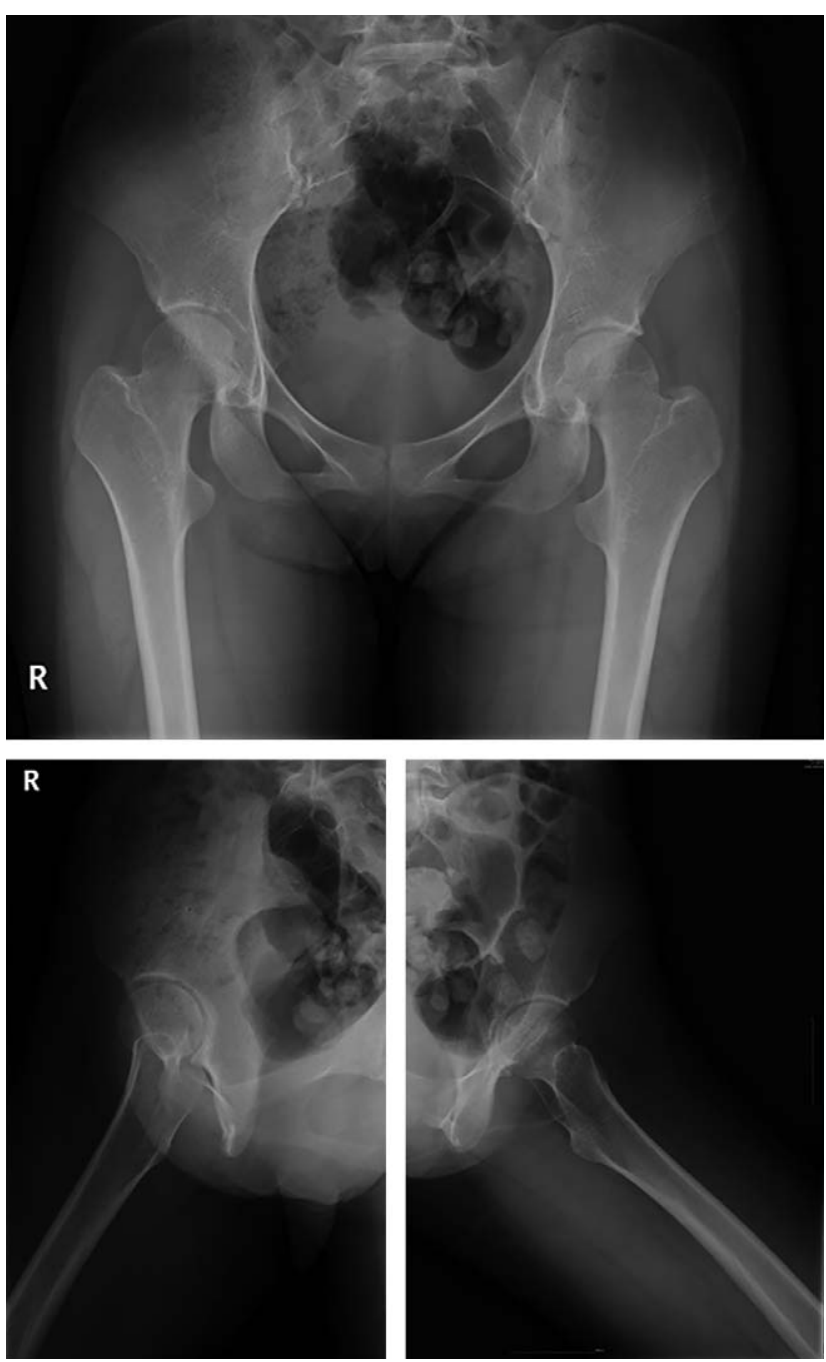

Fig. 1. Both hips anteroposterior and lateral plain X-ray (patient no. 1). 


\section{Hip \& Pelvis}

Joo-Hyoun Song et al. Internal Fixation of Pauwels Type-3 Undisplaced-incomplete Insufficiency Femoral Neck Fractures

which were intracapsular, non-displaced, and requiring surgical fixation, were enrolled. The cohort included those who developed non-specific hip or groin pain preceding the fracture; patients injured with high velocity trauma were excluded.

Mean patient age at time of injury was 61.8 years (range: 20-82 years). Mean duration between initial symptoms and fracture was 12 days (range: 5-21 days). All fractures occurred during normal daily activities. Mean duration of surgery was 60 minutes (range: 38-80 minutes) (Table 1).

Fractures involved three right hips and one left hip. All patients received emergency surgery within 24 hours after hospital admission. Internal fixation was achieved with a cephalomedullary nail (Gamma 3 locking nail system;
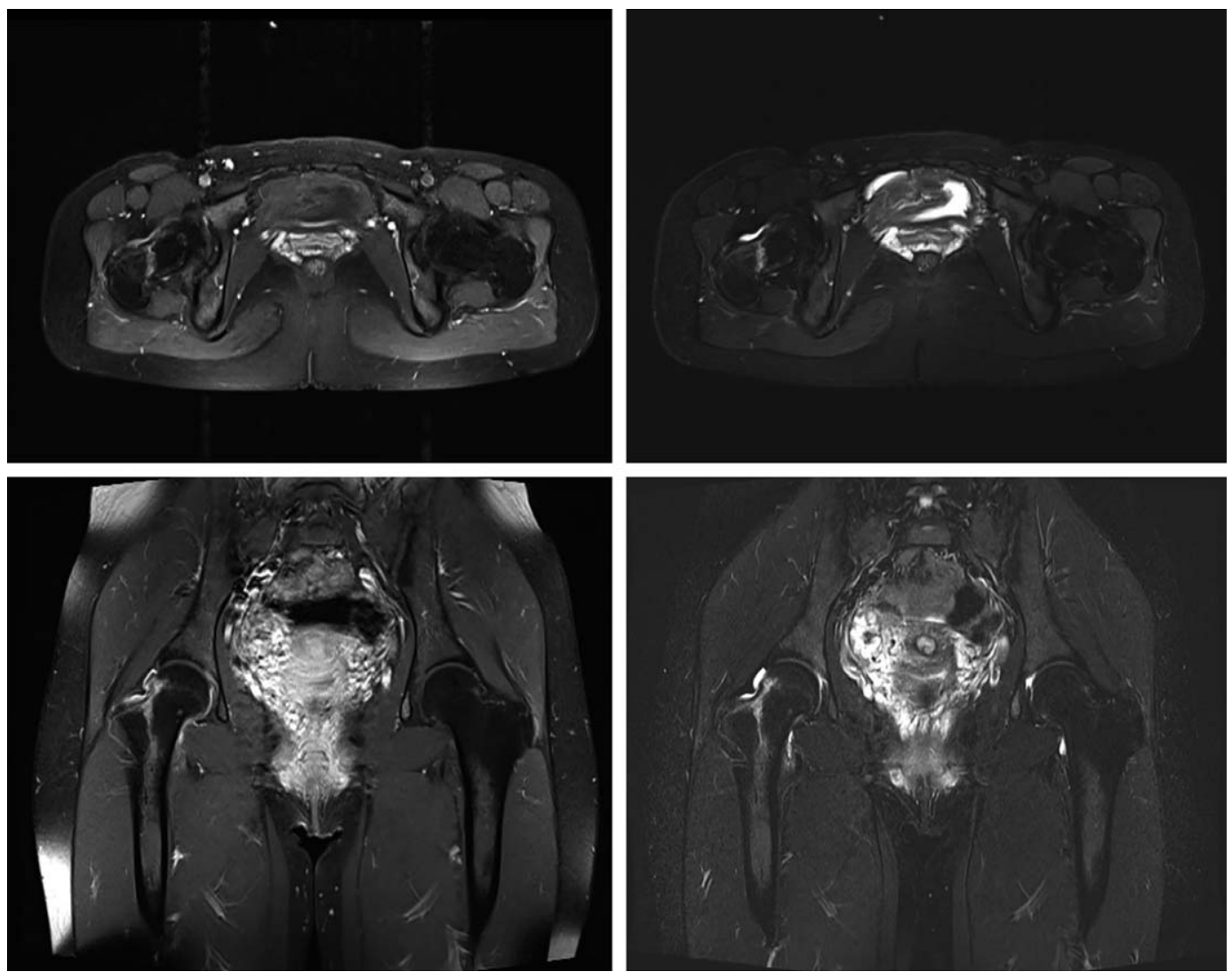

Fig. 2. Magnetic resonance imaging hip (enhanced) Dotarem: T1 contrast-enhanced axial and coronary view, T2 fat suppression axial and coronary view (patient no. 1).

Table 2. Laboratory Test Results

\begin{tabular}{lcc}
\hline \hline Test & Result & Reference value \\
\hline Serum 1, 25-(OH) ${ }_{2}$ vitamin D (pg/mL) & $18.0(13.8-23.6)$ & $19.6-54.3$ \\
Serum 25-OH vitamin D (ng/mL) & $8.6(7.1-11.2)$ & Deficiency: <20.00 \\
Parathyroid hormone-intact (pg/mL) & $151.0(29.9-236.3)$ & $15-53$ \\
Serum calcium (mg/dL) & $8.6(7.6-9.3)$ & $8.4-10.4$ \\
Urine calcium (mg/dL) & $6.4(0.9-11.9)$ & $7.0-18.0$ \\
Serum phosphorus (mg/dL) & $4.0(3.8-4.2)$ & $2.5-4.5$ \\
Urine phosphorus (mg/dL) & $39.7(21.1-58.2)$ & - \\
Calculated 24 hours urine phosphorus (mg/day) & $332.9(316.5-349.2)$ & $400-1,300$ \\
Alkaline phosphatase (IU/L) & $83(63-107)$ & $30-120$ \\
Osteocalcin (ng/mL) & $35.4(17.4-66.4)$ & $11-43$ \\
C-Telopeptide of collagen type 1, $\beta$-crosslink (CTX) (ng/mL) & $0.710(0.380-1.240)$ & $<0.573$ \\
\hline
\end{tabular}

Values are presented as mean (range) or range. 


\section{Hip \& Pelvis}

Hip Pelvis 32(2): 105-111, 2020

Stryker) implant, which consists of a $170 \mathrm{~mm}$ titanium proximal femoral nail to increase fatigue strength either with standard lag screws or with the U-Blade (RC) Lag Screw in the setting of $125^{\circ}$ neck-shaft angle, $35 \mathrm{~mm}$ distal locking screw, set screw and end cap.

Plain radiographs revealed normal findings (Fig. 1), with no fractures, space-occupying lesions, or bony lesions. MRI of the pelvis revealed a unilateral undisplaced-incomplete femoral neck fracture with joint effusion in high shear angle $\left(>70^{\circ}\right)$ (Fig. 2).

The body mass index were all in the normal, healthy range (mean: $21.3 \mathrm{~kg} / \mathrm{m}^{2}$, range: $17.6-27.2 \mathrm{~kg} / \mathrm{m}^{2}$ ). Preoperative laboratory studies revealed adult ostemalacia, with decreased 25-hydroxy-vitamin D (25 (OH) D) and 1, 25dihydroxy-vitamin D (1,25 (OH) 2D) levels. Parathyroid hormone level, serum and urine calcium levels, serum and urine phosphorus, alkaline phosphatase, bone turnover markers C-Telopeptide of collagen type $1, \beta$-crosslink
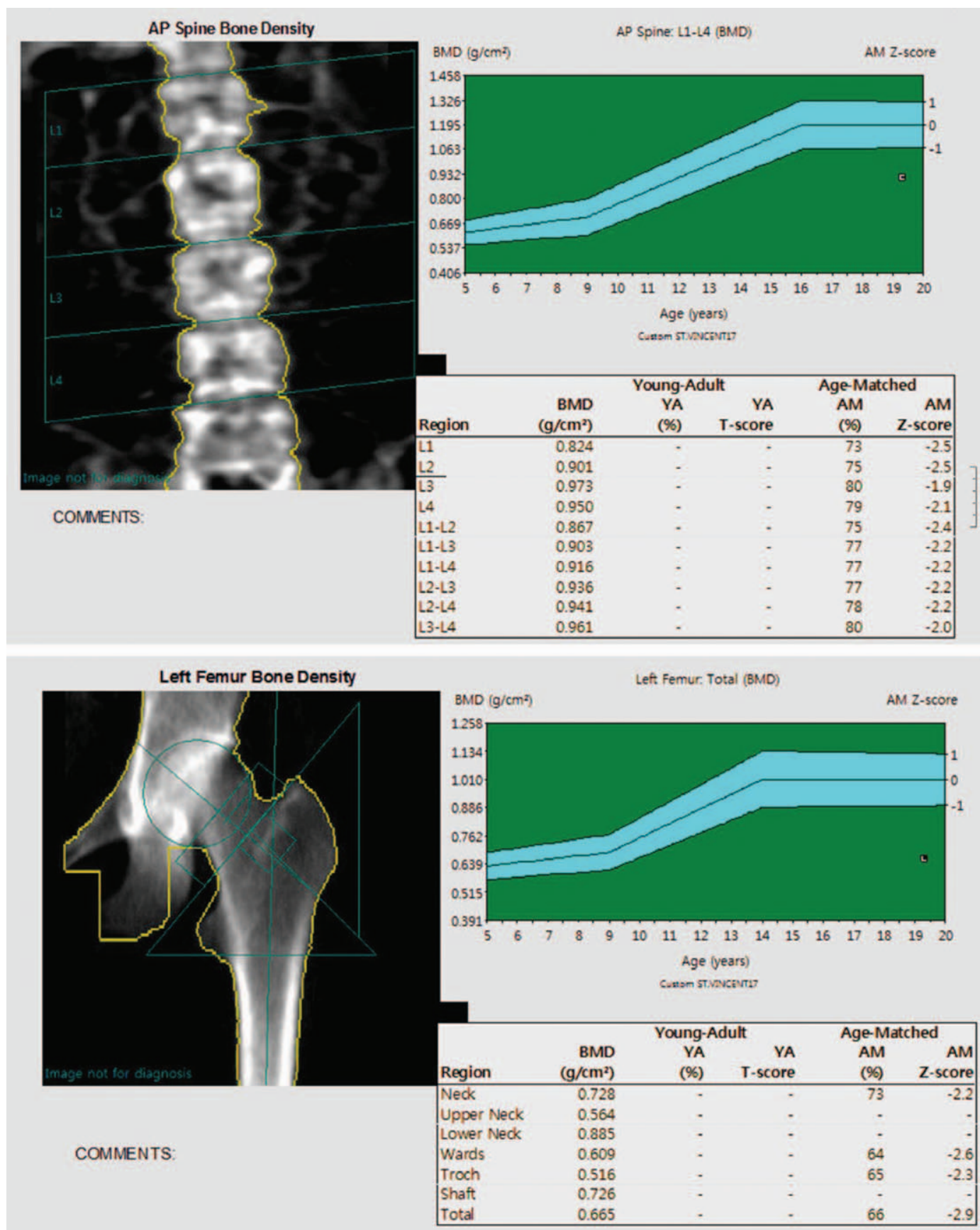

Fig. 3. Bone densitometry, spine/left femur (patient no. 1). 
Joo-Hyoun Song et al. Internal Fixation of Pauwels Type-3 Undisplaced-incomplete Insufficiency Femoral Neck Fractures

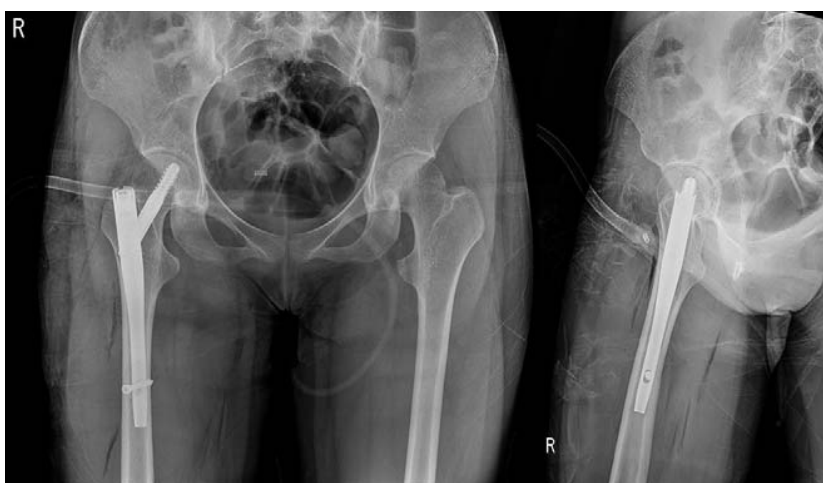

Fig. 4. Postoperative both hips anteroposterior and right lateral plain X-ray (patient no. 1).

(CTX), N-Telopeptide of collagen type 1 (NTX), and osteocalcin were all normal (Table 2). Low BMD was observed using dual-energy X-ray absorptiometry (DEXA) (Fig. 3). All patients were diagnosed, via serum tests, with adult osteomalacia before the operation.

All patients underwent osteosynthesis with cephalomedullary nails under general anesthesia (Fig. 4). Partial weight bearing was allowed just after the surgery for six weeks. Patients indicated that they were free of pain and full weight bearing was achieved after six weeks. Adequate vitamin D, iron replacement and regular diet were provided.

After the operation, a whole body bone scan was done to evaluate the underlying stress fracture. No other definite abnormal uptake except for the unilateral hip joint were observed in any patient (Fig. 5).

All patients achieved good results, with full range of motion, no pain, no complications and a return to normal funtion. The average Harris hip score increased to 89.5 at six months postoperatively. Patients were followed up for a minimum of one year after surgery (range: 12 months to 3 years) and all of had radiographs at postoperatively one year that demonstrated solid fracture healing.

\section{DISCUSSION}

In this study, four consecutive patients with unilateral Pauwels type-3 vertical femoral neck undisplacedincomplete insufficiency fractures secondary to osteomalacia are presented.

A general laborer with an atraumatic femoral neck insufficiency fracture is unusual; only a few cases associated with specific medical conditions have been reported. Similar to our series, there are reports associated with osteomalacia secondary to vitamin D deficiency or inadequate sun exposure. Another case was reportedly due to smoking and alcohol-abuse induced osteoporosis ${ }^{7}$. Several other reports correlating this fracture type to a metabolic disease can also be found. Therefore, early suspicion and consideration in an unexpected population is highly important in suspected stress cases. Delayed detection is associated with complete displacement of the femoral head and may precipitate femoral non-union or avascular necrosis, resulting in long-term functional deficit.

Second, patients were subsequently diagnosed with osteomalacia based on DEXA Z-score and laboratory test results. Osteomalacia is one of the most common causes of insufficiency stress fractures. It is a bone metabolism disorder associated with defective bone mineralization, which causes softening of the bones and potential for stress fractures occurring at typical sites of weight-bearing bones (e.g., femur). The underlying cause of osteomalacia is usually vitamin D deficiency; there are several risk factors for these fractures, including osteomalacia. Vitamin D deficiency is a worldwide problem that may result from: i) inadequate intake and limited sunlight exposure, or ii) inadequate vitamin $\mathrm{D}$ absorption and abnormal vitamin D metabolism with multiple causes including liver or kidney disease. There are several reports of femoral neck insufficiency fractures due to vitamin $\mathrm{D}$ deficiency ${ }^{8}$. One study in UK reported that more than $50 \%$ of the adult population had too little vitamin D and $16 \%$ had a severe deficiency during winter and spring ${ }^{9}$. Therefore, an accurate history and analysis of serum vitamin D levels must be considered for evaluation.

These cases were noteworthy because fixation was conducted using a cephalomedullary nail (Gamma3 locking nail system; Stryker). Femoral neck stress fractures may be classified as compressive or tensile type ${ }^{10)}$, the later has a fracture line from the superior border, which can lead to displacement and require surgical fixation. Established treatment conventions recommend that if there is more than $50 \%$ involvement of the compression side of the femoral neck or any aspect of the tension side, internal fixation should be performed. In these cases, all patients had tensile-type femoral neck fractures. Surgical treatment options include cannulated screws, dynamic hip screws, proximal femoral nails, and angled or anatomic plates. Successful treatments using dynamic hip screws were reported in several articles, however, treatment using cephalomedullary nails has not been reported. Cephalomedullary nails can also be used as a safe alternative to multiple parallel screws or a sliding 


\section{Hip \& Pelvis}

Hip Pelvis 32(2): 105-111, 2020

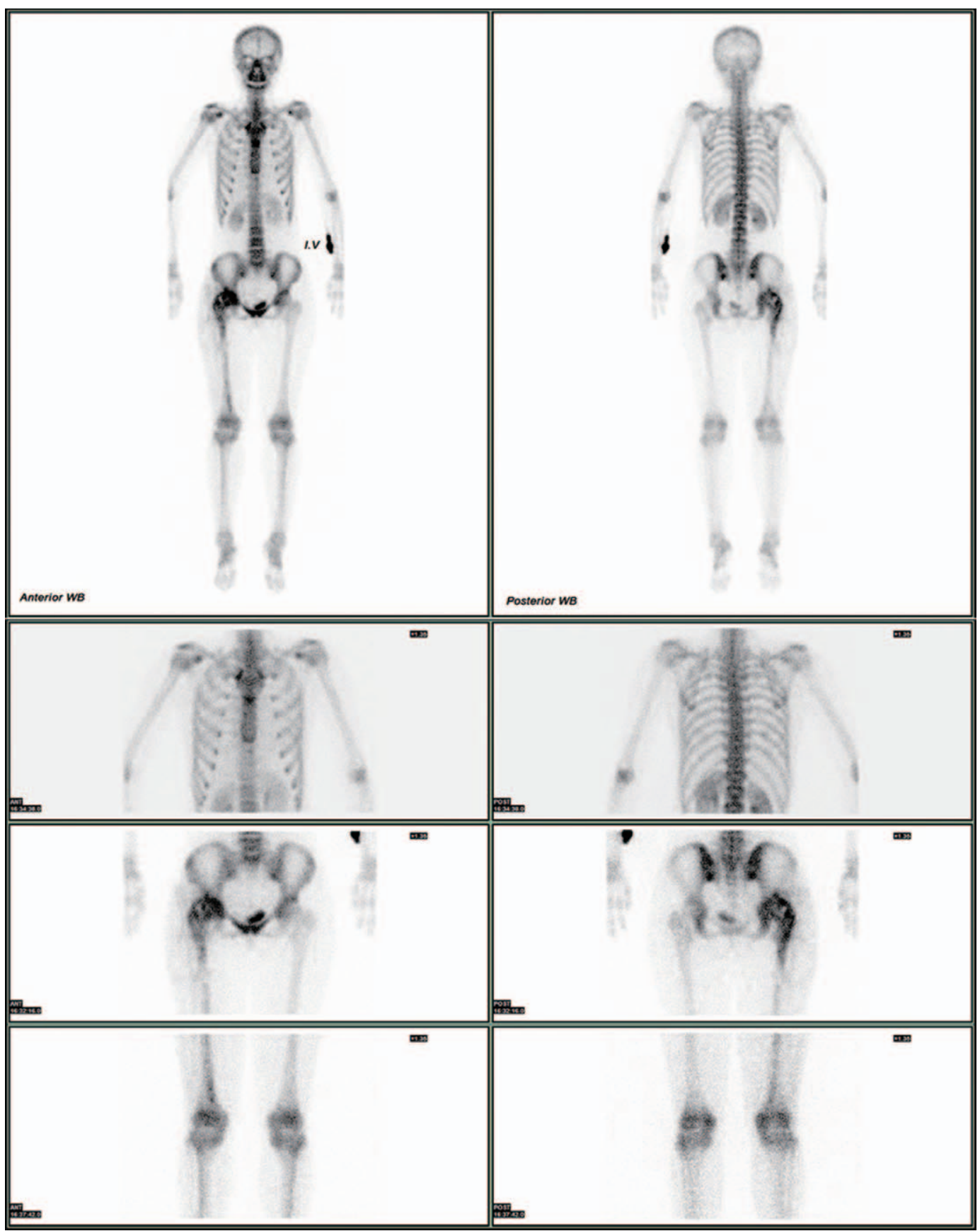

Fig. 5. Postoperative whole-body Tc-99 m hydroxymethylene diphosphonate bone scan (patient no. 1).

hip screw for fixation of femoral neck stress fractures. In the patients described here, Pauwels type-3 femoral neck fractures tend to be more unstable and associated with more complications. A biochemical study revealed that the stiffness of fixed-angle devices are superior to cannulated screws alone for the fixation of a Pauwels type-3 fracture in cadaveric femora ${ }^{5}$. In one clinical study, treatment with a fixed-angle device, such as a cephalomedullary nail, led to less nonunion and osteonecrosis, although these results did not achieve significance ${ }^{6}$.

When planning surgery, there are several studies evaluating outcomes of various internal fixation strategies for femoral neck fractures. However, since femoral neck stress fractures are rare themselves, no study exists to evaluate internal fixation methods for their treatment. We chose the implant type based on the studies for general femoral neck fractures. Also, this fracture pattern is rarely encountered, making it difficult to obtain a large enough series to study. With 


\section{Hip \& Pelvis}

Joo-Hyoun Song et al. Internal Fixation of Pauwels Type-3 Undisplaced-incomplete Insufficiency Femoral Neck Fractures

such a type of fracture with more vertical orientation, a cephalomedullary nail could be a safe alternative for internal fixation. More studies, with larger series comparing outcomes of various implant types will help better elucidate the most appropriate surgical approach for this rare fracture.

\section{CONFLICT OF INTEREST}

The authors declare that there is no potential conflict of interest relevant to this article.

\section{REFERENCES}

1. Wentz L, Liu PY, Haymes E, Ilich JZ. Females have a greater incidence of stress fractures than males in both military and athletic populations: a systemic review. Mil Med. 2011; 176:420-30.

2. Steele CE, Cochran G, Renninger C, Deafenbaugh B, Kuhn KM. Femoral neck stress fractures: MRI risk factors for progression. J Bone Joint Surg Am. 2018;100:1496-502.

3. Aaron JE, Gallagher JC, Anderson J, et al. Frequency of osteomalacia and osteoporosis in fractures of the proximal femur. Lancet. 1974;1:229-33.

4. De Marchi J, Bajwa R, Duggan SN, Magill P. Suspected coexistent osteoporosis and osteomalacia with atraumatic bilateral neck of femur fractures in a 53-year-old man without apparent risk factors. BMJ Case Rep. 2014;2014:bcr2014205205.

5. Johnson JP, Borenstein TR, Waryasz GR, et al. Vertically oriented femoral neck fractures: a biomechanical comparison of 3 fixation constructs. J Orthop Trauma. 2017;31:363-8.

6. Liporace F, Gaines R, Collinge C, Haidukewych GJ. Results of internal fixation of Pauwels type-3 vertical femoral neck fractures. J Bone Joint Surg Am. 2008;90:1654-9.

7. Fukui K, Kaneuji A, Hirata H, et al. Bilateral spontaneous simultaneous femoral neck occult fracture in a middle-aged man due to osteoporosis and vitamin D deficiency osteomalacia: a case report and literature review. Int J Surg Case Rep. 2019;60:358-62.

8. Nagao S, Ito K, Nakamura I. Spontaneous bilateral femoral neck fractures associated with a low serum level of vitamin D in a young adult. J Arthroplasty. 2009;24:322.e1-4.

9. Pearce SH, Cheetham TD. Diagnosis and management of vitamin D deficiency. BMJ. 2010;340:b5664.

10. Fullerton LR Jr, Snowdy HA. Femoral neck stress fractures. Am J Sports Med. 1988;16:365-77. 